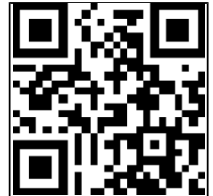

Editor's choice Scan to access $m$ free conten

${ }^{1}$ Department of Physiotherapy, School of Primary Health Care, Monash University, Melbourne, Victoria, Australia

${ }^{2}$ Department of Rehabilitation, Nutrition and Sport, School of Allied Health, La Trobe University, Melbourne, Victoria, Australia

${ }^{3}$ Department of Physical Therapies, Australian Institute of Sport, Bruce, Australian Capital Territory, Australia

${ }^{4}$ University of Canberra, Canberra, Australian Capital Territory, Australia ${ }^{5}$ Sansom Institute for Health Research, University of South Australia \& Pain Adelaide, Adelaide, South Australia, Australia

${ }^{6}$ Cognitive Neuroscience Unit, Deakin University, Burwood, Victoria, Australia

\section{Correspondence to}

Ebonie Rio, Department of Physiotherapy, School of Primary Health Care, Monash University, Peninsula Campus, P.O. Box 527, Frankston, Melbourne, VIC 3199, Australia; ebonie.rio@monash. edu

Accepted 20 April 2015 Published Online First 15 May 2015

\title{
Isometric exercise induces analgesia and reduces inhibition in patellar tendinopathy
}

\author{
Ebonie Rio, ${ }^{1}$ Dawson Kidgell, ${ }^{2}$ Craig Purdam, ${ }^{3}$ Jamie Gaida, ${ }^{1,4} \mathrm{G}$ Lorimer Moseley, \\ Alan J Pearce, ${ }^{6}$ Jill Cook'
}

\section{ABSTRACT}

Background Few interventions reduce patellar tendinopathy (PT) pain in the short term. Eccentric exercises are painful and have limited effectiveness during the competitive season. Isometric and isotonic muscle contractions may have an immediate effect on PT pain.

Methods This single-blinded, randomised cross-over study compared immediate and 45 min effects following a bout of isometric and isotonic muscle contractions. Outcome measures were PT pain during the single-leg decline squat (SLDS, 0-10), quadriceps strength on maximal voluntary isometric contraction (MVIC), and measures of corticospinal excitability and inhibition. Data were analysed using a split-plot in time-repeated measures analysis of variance (ANOVA).

Results 6 volleyball players with PT participated. Condition effects were detected with greater pain relief immediately from isometric contractions: isometric contractions reduced SLDS (mean \pm SD) from 7.0 \pm 2.04 to $0.17 \pm 0.41$, and isotonic contractions reduced SLDS (mean $\pm S D$ ) from $6.33 \pm 2.80$ to $3.75 \pm 3.28$ ( $p<0.001$ ). Isometric contractions released cortical inhibition (ratio mean \pm SD) from $27.53 \% \pm 8.30$ to $54.95 \% \pm 5.47$, but isotonic contractions had no significant effect on inhibition (pre $30.26 \pm 3.89$, post $31.92 \pm 4.67$; $p=0.004$ ). Condition by time analysis showed pain reduction was sustained at 45 min postisometric but not isotonic condition $(p<0.001)$. The mean reduction in pain scores postisometric was 6.8/10 compared with 2.6/10 postisotonic. MVIC increased significantly following the isometric condition by $18.7 \pm 7.8 \%$, and was significantly higher than baseline $(p<0.001)$ and isotonic condition $(p<0.001)$, and at $45 \mathrm{~min}(p<0.001)$. Conclusions $A$ single resistance training bout of isometric contractions reduced tendon pain immediately for at least 45 min postintervention and increased MVIC. The reduction in pain was paralleled by a reduction in cortical inhibition, providing insight into potential mechanisms. Isometric contractions can be completed without pain for people with PT. The clinical implications are that isometric muscle contractions may be used to reduce pain in people with PT without a reduction in muscle strength.

\section{INTRODUCTION}

Tendinopathy (tendon pain and dysfunction) in athletes is difficult to manage. Eccentric exercise, which is the most commonly prescribed exercise for the treatment of tendinopathy, ${ }^{1-4}$ is often painful to complete. ${ }^{5}$ Tendinopathy is especially problematic in the competitive season, when there are constant time and performance pressures. ${ }^{6}$ Where eccentric exercise has been completed in the competitive season, there has been poor adherence due to increased pain, and either no benefit ${ }^{7}$ or worse outcomes. ${ }^{8}$ Athletes are reluctant to cease sporting activity to complete eccentric exercise programmes ${ }^{9}$ and they may be more compliant with exercise strategies that reduce pain to enable ongoing sports participation.

Exercise-induced pain relief would have several clinical benefits. First, athletes may be able to manage their pain with exercises either immediately prior to or following activity. Second, exercise is non-invasive and without potential pharmacological side effects or sequelae of long-term use that are associated with some interventions. Third, exercises that reduce pain are likely to have greater adherence. Therefore, alternative muscle contraction types, other than eccentric exercises, warrant investigation.

Isotonic exercise (heavy, slow, concentric and eccentric resistance training) has been shown to be as effective as eccentric only exercise in patellar tendinopathy (PT) for tendon pain and activity participation; ${ }^{1}{ }^{10}$ however, the immediate effect of isotonic exercise on pain has not been studied. Isometric muscle contractions have been shown to reduce pressure pain thresholds in normal participants, ${ }^{11}{ }^{12}$ but have not been investigated in tendon pain. The pain inhibition following a local isometric contraction, demonstrated in previous studies of normal participants, is widespread; ${ }^{12}$ this indicates central nervous system (CNS) involvement and warrants investigation.

The effect of exercise on the motor cortex may be modulated in the presence of pain. Exercises that are painful to complete may change motor control and cause cortical reorganisation, as pain itself is known to alter cortical representation. ${ }^{13}$

This may contribute to persistence of tendon pain through the continuation of aberrant motor patterns. In the CNS, the primary regions involved in motor control are the primary motor cortex and corticospinal tract, which activate the motor neurone pool and control motor function. Changes in motor output, however, are a combination of changes in the excitatory and inhibitory neural pathways. This motor neuroaxis can be investigated using single-pulse transcranial magnetic stimulation (TMS). Paired-pulse TMS can measure short-interval intracortical inhibition (SICI), which is thought to be mediated at a cortical level via $\mathrm{GABA}_{\mathrm{A}}$ receptors ${ }^{14}$ rather than at the spinal cord and quantifies the effect of the inhibitory neurons that synapse onto pyramidal cells in the primary motor cortex. ${ }^{15}$

Exercise, the cornerstone of tendon rehabilitation, is capable of changing excitatory and inhibitory measures. ${ }^{16}{ }^{17}$ No study has examined if 
exercise can immediately reduce tendon pain and if exercise changes the motor neuroaxis. This may clarify the mechanism behind clinical improvement following exercise-based rehabilitation. $^{18}$

The primary aim of this study was to determine if either isotonic or isometric exercise would induce immediate pain relief in PT. The secondary aim was to explore the mechanisms and investigate changes to cortical motor function. Therefore, this study compared an acute bout of isometric and isotonic quadriceps loading on patellar tendon pain, maximal voluntary isometric strength and measures of corticospinal excitability and inhibition.

\section{METHOD}

This was a within respondents, single-blinded, randomised cross-over trial with two intervention arms (figure 1). Six male volleyball athletes (median age 26.9 years, range 18-40 years) who were taking no medication were recruited for the study. Three had unilateral pain and three had bilateral patellar tendon pain. This study was approved by university ethics committees and all athletes provided written informed consent. Diagnosis of PT was made by the same experienced sports physiotherapist on clinical presentation-pain localised to the inferior pole of the patella during jumping and landing activities, and during testing on the single-leg decline squat (SLDS). The diagnosis was confirmed by the presence of characteristic features on ultrasound imaging (eg, hypoechoic area). All athletes were playing once per week and training twice per week. The TMS tester was blind to intervention status during testing and all data were analysed blind to intervention status. All testing was completed exactly 1 week apart to ensure stability of loading prior to testing (training and game days were consistent). The order of intervention was randomised by asking the athlete to draw an opaque sealed envelope with no external markings (concealed randomisation). ${ }^{19}$

\section{Baseline testing}

At week 1, baseline testing was completed without any intervention to ensure that the strength and pain testing measures and TMS measures themselves did not affect the primary outcome measures and to familiarise participants with the equipment and protocol. Athletes with bilateral symptoms were asked to nominate their most painful knee on the SLDS and measures of quadriceps torque were taken from this side only; the contralateral hemisphere was tested with TMS. Athletes were asked to complete a Victorian Institute of Sport Assessment-patellar tendon (VISA-P), a questionnaire about patellar tendon pain and function that is scored between 0 and 100, with 100 being maximal pain free function. ${ }^{20}$ At weeks 2 and 3 , baseline measures were repeated (to enable comparison to week 1 baseline as well as the baseline of that intervention session).

\section{Pain and strength testing}

Tendon pain and quadriceps strength were tested at baseline. The SLDS, a reliable patellar tendon pain provocation test, ${ }^{21}$ was completed on each leg. Participants provided a numerical pain rating score for the decline squat on an 11-point numerical rating scale (NRS), anchored at left with ' 0 , no pain' and at right with '10, worst possible pain'. Maximal voluntary isometric contraction (MVIC) torque for the quadriceps on the tested side was recorded in $\mathrm{Nm}$ using isokinetic equipment (Biodex system 4 Pro, 1 Biodex Medical 2 Systems) with three efforts completed with identical instructions, vocal encouragement and set up for each trial at $60^{\circ}$ of knee flexion. The outcome was the maximum peak torque recorded during these three efforts.

\section{TMS and electromyography}

Baseline measures of corticospinal excitability and SICI were obtained using single-pulse and paired-pulse TMS, respectively. We optimised the accuracy of TMS by aligning the coil with reference markers on a tight fitting cap worn by participants and marked with a latitude-longitude matrix, positioned with reference to the nasion-inion and interaural lines. ${ }^{22}$

Single-pulse stimulus-response curves were obtained during low-level isometric contractions of the quadriceps muscle group. Low-level contractions were performed by maintaining the knee joint at $60^{\circ}$ of flexion while performing a 10\% MVIC, which equated to $10 \pm 2 \%$ of root mean square (RMS) electromyography (rmsEMG) during MVIC (table 1). A 10\% isometric contraction is a non-painful task for someone with PT. Consistent muscle activation was confirmed by recording prestimulus rmsEMG throughout the session. For a single stimulus-response curve, 10 stimuli were delivered in $20 \%$ increments from $90 \%$ of the participant's active motor threshold (AMT) up to $170 \%$ of their AMT. The stimuli were delivered using a ramped protocol. $^{23}$ The amplitude of the motor-evoked potential (MEP) or slope of the stimulus-response curve represents corticospinal excitability. ${ }^{24}$

SICI is a subthreshold stimulus that activates inhibitory interneurons that synapse onto pyramidal neurons in the M1 and results in reduced number of action potentials by the subsequent suprathreshold stimulus. ${ }^{25}$ To quantify SICI, 10 single-pulse stimuli and 10 short-interval paired-pulse stimuli were delivered in random counterbalanced order. Intensity was set at $120 \%$ of AMT, which was determined during familiarisation and adjusted if there was a change in AMT. The conditioning stimulus for paired-pulse stimulation was set at $80 \%$ of AMT, the interstimulus interval was $3 \mathrm{~ms}$, and posterior to anterior current flow was used to induce I3 waves. $^{26} 27$ Muscle activation during testing does not affect $\mathrm{SICI}^{27} \mathrm{SICI}$ is represented as a percentage ratio where high levels of inhibition are indicated by a low SICI ratio. Therefore, an increase in the SICI percentage represents a reduction in inhibition.

Participants were tested at the same time of day and the same day of the week. ${ }^{28}$ TMS was delivered using two Magstim $200^{2}$ stimulators connected via a Bistim unit (Magstim Co, Dyfed, $\mathrm{UK}$ ) and a $110 \mathrm{~mm}$ concave double-cone coil (maximum output of $1.4 \mathrm{~T}$ ). The motor hotspot for the rectus femoris (RF) muscle (with posterior-induced to anterior-induced current flow in the cortex) was determined and AMT were established as the intensity at which at least 5 of 10 stimuli produced MEP amplitudes of greater than $200 \mu \mathrm{V}$ recorded from the RF muscle. The RF muscle was selected as the best representative muscle as the tendon fibres of the RF muscle are the only tendon fibres of the quadriceps muscle group that continue over the anterior surface of the patellar to form the patellar tendon, ${ }^{29}$ which was the tendon of interest. Valid EMG data for RF can be captured with surface electrodes.

The area of electrode placement was shaved to remove fine hair, rubbed with an abrasive skin rasp to remove dead skin, and then cleaned with 70\% isopropyl alcohol. Bipolar gel $\mathrm{Ag}-\mathrm{AgCl}$ electrodes (8 mm diameter, model E258S; Biopac, Goleta, California, USA) were placed over the RF muscle (centre-centre interelectrode distance $=2 \mathrm{~cm}$ ). A grounding electrode was placed over the patella and subsequently, used as a common reference for all electrodes. All cables were fastened with tape to prevent movement artefact. The location of electrodes were 
Figure 1 Testing protocol of weeks 2 and 3 (AMT, active motor threshold; CON, concentric phase; CS, conditioning stimulus; ECC, eccentric phase; MVIC, maximal voluntary isometric contraction; RM, repetition maximum; $\mathrm{SICl}$, short-interval intracortical inhibition; SLDS, single-leg decline squat; TMS, transcranial magnetic stimulation; TS, test stimulus).

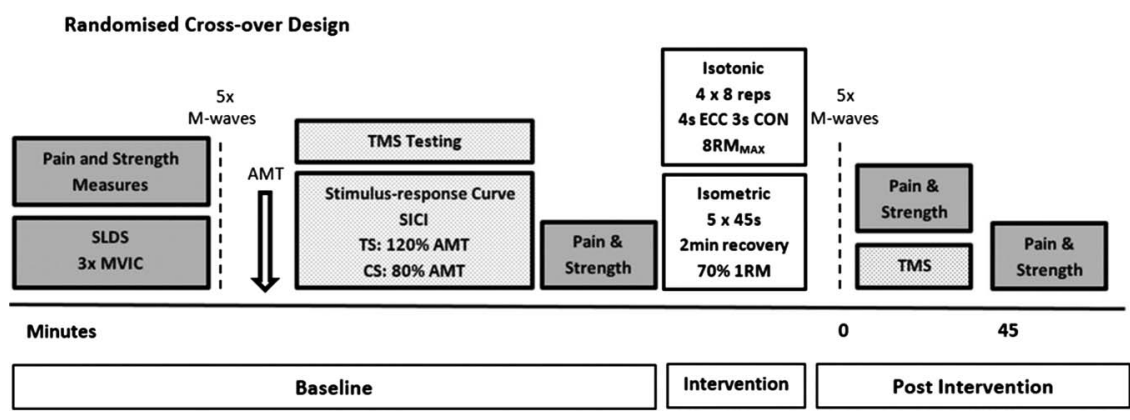

marked with a permanent marker by tracing around the electrode, and measured from anterior inferior iliac spine and patella to maximise consistency of electrode placement relative to the innervation zone. An impedance metre was used to check that impedance did not exceed $10 \mathrm{k} \Omega$ prior to testing. Surface EMG $($ sEMG) signals were amplified $(\times 1000)$, bandpass filtered (high pass at $13 \mathrm{~Hz}$, low pass at $1000 \mathrm{~Hz}$ ), digitised online at $2 \mathrm{kHz}$ for $500 \mathrm{~ms}$, recorded and analysed using PowerLab 4/35 (ADInstruments, Bella Vista, Australia).

Prestimulus rmsEMG activity was determined in the RF muscle, $100 \mathrm{~ms}$ prior to each TMS stimulus during pretesting and post-testing. Any trial in which prestimulus rmsEMG exceeded $10 \pm 2 \%$ of maximal rmsEMG was discarded and the trial repeated. The surface rmsEMG was calculated from a $500 \mathrm{~ms}$ segment that occurred during the asymptote of each maximal voluntary contraction (MVC) and was calculated as the amplitude of the RMS value.

\section{Maximal compound muscle action potential}

Direct muscle responses were obtained from the RF muscle by supramaximal electrical stimulation (pulse width $2000 \mu \mathrm{s}$; DS7A, Digitimer, UK) of the femoral nerve under resting conditions. The site of stimulation that produced the largest $\mathrm{M}$-wave was located by positioning the bipolar electrodes in the femoral triangle. An increase in current strength was applied to the femoral nerve until there was no further increase observed in the amplitude of the sEMG response $\left(\mathrm{M}_{\mathrm{MAX}}\right)$. To ensure $\mathrm{M}_{\max }$ was obtained, the current was increased an additional $20 \%$ for five more stimuli, with a period of $6-9$ s separating each stimulus to ensure there were no further increases. $\mathrm{M}_{\mathrm{MAX}}$ was recorded at baseline and following the intervention, to ensure that there were no changes in peripheral muscle excitability that could influence MEP amplitude. ${ }^{30}$

\section{Intervention protocols}

Protocols were matched for time under load and rest between sets (set at 2 min to allow muscle recovery) $)^{31}$ (table 2). Repetition maximum and MVIC were determined in the familiarisation session. As muscle work during isometric exercise and isotonic exercise cannot be directly measured, protocols were matched for perceived exertion on the basis of pilot studies.
Isotonic repetitions were paced by a metronome. The metronome was also used for isometric contractions to control for any potential confounding effect. Furthermore, auditory cues have been shown to be beneficial on the induction of neuroplasticity. $^{16} 32-36$

\section{Follow-up testing and time course}

Testing immediately after the intervention consisted of pain and strength testing measures (SLDS and MVIC), $\mathrm{M}_{\text {MAX }}$ and TMS measures (stimulus-response curve and SICI). Testing for $\mathrm{M}_{\mathrm{MAX}}$ was timed so that it was tested a minimum of 4-5 min after training. ${ }^{37} 38$ SLDS and MVIC were also tested 45 min postintervention (figure 1 ).

\section{Data analyses}

The peak-to-peak amplitude of MEPs evoked in the period 10$50 \mathrm{~ms}$ after stimulation were analysed using LabChart 8 software (ADInstruments, Bella Vista, New South Wales, Australia) after each stimulus was automatically flagged with a cursor, providing peak-to-peak values in $\mu \mathrm{V}$, averaged and normalised to the $\mathrm{M}_{\max }$ and multiplied by 100 . To construct stimulus-response curves, stimulus intensity was plotted against MEP amplitude and then fitted with a non-linear Boltzmann equation using Prism 6 (Graphpad software Inc, California, USA). ${ }^{36}$

The conditioning MEP amplitude was expressed as a percentage of the unconditioned test MEP amplitude to calculate the level of intracortical inhibition.

\section{Statistical analysis}

All data were screened with the Brown-Forsythe test and found to be normally distributed (all p $>0.05$ ) and thus, the assumptions of the analysis of variance (ANOVA) were not violated. A split-plot in time-repeated measures ANOVA was used to compare the effect of each condition (isometric and isotonic) on pain, strength, and corticospinal excitability and inhibition. When appropriate, univariate and post-hoc $t$ tests (with Bonferroni correction) analyses for pairwise comparisons of means for each dependent measure were used when significant interactions were found. For all tests, if the assumption of sphericity was violated, the Huynh-Feldt correction was applied. The $\alpha$ was set at $\mathrm{p}<0.05$; all results are displayed as means \pm SD and

Table 1 Means $( \pm$ SEM) root mean square EMG: condition average as a percentage of EMG recorded during maximal isometric contraction

\begin{tabular}{|c|c|c|c|c|c|c|}
\hline \multirow[b]{2}{*}{ Exercise } & \multicolumn{2}{|c|}{ SP $130 \%$ AMT } & \multicolumn{2}{|c|}{ SP $120 \%$ AMT } & \multicolumn{2}{|l|}{ PP } \\
\hline & Pre & Post & Pre & Post & Pre & Post \\
\hline Isotonic trials & $10.80 \pm 0.15$ & $10.69 \pm 0.16$ & $10.34 \pm 0.18$ & $10.25 \pm 0.17$ & $10.77 \pm 0.16$ & $10.69 \pm 0.16$ \\
\hline Isometric trials & $10.42 \pm 0.11$ & $10.67 \pm 0.10$ & $10.63 \pm 0.16$ & $10.69 \pm 0.12$ & $10.47 \pm 0.13$ & $10.25 \pm 0.15$ \\
\hline
\end{tabular}


Table 2 Loading protocols in the study

\begin{tabular}{|c|c|c|c|c|}
\hline & Apparatus & Prescription & $\begin{array}{l}\text { Recovery } \\
\text { (min) }\end{array}$ & $\begin{array}{l}\text { Loading } \\
\text { bolus }\end{array}$ \\
\hline Isometric & Biodex Pro & $5 \times 45 \mathrm{~s}$ at $60^{\circ}$ & 2 & $70 \% \mathrm{MVC}$ \\
\hline Isotonic & $\begin{array}{l}\text { Leg } \\
\text { extension } \\
\text { machine }\end{array}$ & $\begin{array}{l}4 \times 8 \text { repetitions } \\
4 \mathrm{~s} \text { eccentric } \\
\text { phase } \\
3 \mathrm{~s} \text { concentric } \\
\text { phase }\end{array}$ & 2 & $100 \% 8 \mathrm{RM}$ \\
\hline
\end{tabular}

CI, where appropriate, within the text. Where mean \pm SEM are reported, this has been indicated.

\section{RESULTS}

Participants had substantial tendon pain, the mean VISA-P was 52.8 (47.5 to 66.5). There were no systematic differences detected in baseline SLDS pain, stimulus-response slope, SICI or MVIC at the first or each subsequent testing session indicating sufficient wash-out between sessions. Therefore, only preintervention and postintervention data (weeks 2 and 3) are reported. There were no differences detected in $\mathrm{M}_{\mathrm{MAX}}$ at any time point $(p=0.88)$. The $10 \%$ muscle contraction maintained during TMS testing was a pain-free activity for participants.

Baseline SLDS pain did not differ significantly prior to either intervention. Preisometric intervention pain (mean \pm SD) was $7 /$ $10 \pm 2.04$ and preisotonic intervention pain was $6.33 / 10 \pm 2.80$ $(p>0.99)$. Isometric exercise reduced pain on the SLDS (mean $\pm S D)$ immediately from $7 / 10 \pm 2.04$ to $0.17 / 10 \pm 0.41$ $(p=0.004)$; the reduction was sustained at $45 \mathrm{~min}(\mathrm{p}<0.001$; figure 2). Isotonic exercise resulted in immediate pain relief on SLDS (mean \pm SD) from $6.33 / 10 \pm 2.80$ to $3.75 / 10 \pm 4.67$ $(p=0.04)$, but this was not sustained at $45 \mathrm{~min}$. This corresponds to an immediate mean reduction in pain following

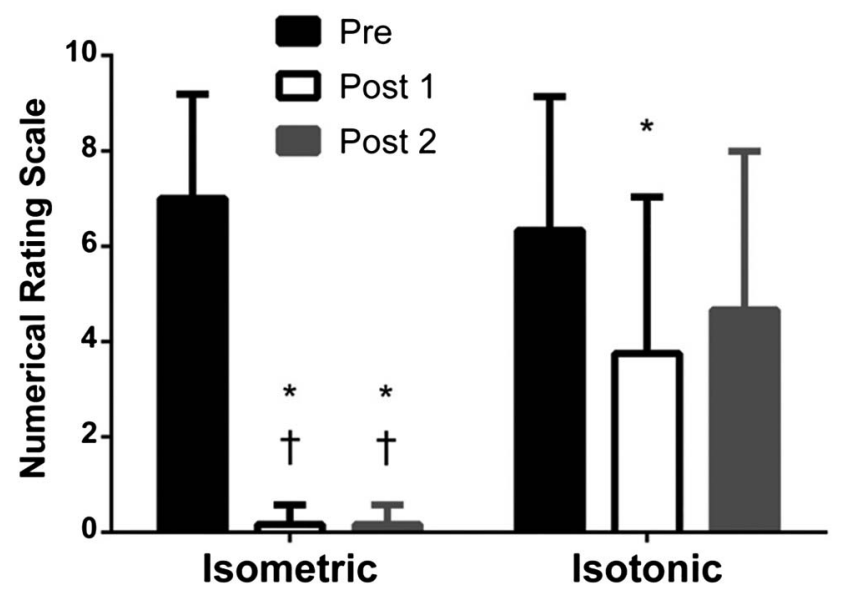

Figure 2 Single-leg decline squat (SLDS; tendon pain) preintervention and postintervention: mean $( \pm S D)$ changes in SLDS preisometric and postisometric and preisotonic and postisotonic strength training. Immediately following an acute bout of isometric strength training, SLDS numerical pain rating scale improved by $87 \%$ and was sustained at 45 min post compared with isotonic. Immediately following an acute bout of isotonic strength training, SLDS numerical pain rating scale improved by $42 \%$, however by 45 min post the intervention, it was not significantly different to baseline. * Denotes difference to preintervention $(p<0.05) ; \dagger$ denotes difference to same time point for the isotonic intervention $(p<0.05)$. isometric exercise of $6.8 / 10$ compared with $2.6 / 10$ postisotonic exercise. Individual data are shown in figure 3.

An increased MVIC torque was observed immediately after the isometric intervention (mean increase of $18.7 \%$ compared with baseline isometric, mean difference between isometric and isotonic $27 \mathrm{Nm}, 95 \% \mathrm{CI} 12.96$ to $41.04, \mathrm{p}<0.001)$ that was sustained for at least $45 \mathrm{~min}$ postintervention (mean increase from isometric baseline $17.4 \%$, mean difference between isometric and isotonic $30.5 \mathrm{Nm}, 95 \%$ CI 16.46 to 44.54 , $\mathrm{p}<0.001)$. This increase was significantly different to the isotonic exercise that resulted in a small, but non-significant reduction in MVIC immediately following the intervention and at 45 min after (figure 4).

The mean SICI ratio prior to the isometric intervention was $27.53 \pm 8.30 \%$ (figure 5), and prior to the isotonic condition was $30.26 \pm 3.89 \%(p=0.31)$. There was a significant condition by time effect immediately postintervention; isometric exercise significantly released this inhibition to $54.95 \pm 5.47 \%$ (an increase in SICI ratio; mean difference compared with isotonic 23.66, 95\% CI 12.28 to $35.05, \mathrm{p}=0.004$ ) (figure 5). Inhibition was not measured at $45 \mathrm{~min}$.

There was no difference in SLDS pain scores in the contralateral leg after a unilateral exercise intervention in people with bilateral PT; however, there were only three participants. There were no systematic differences detected between isometric and isotonic exercise in corticospinal excitability, as represented by the slope of the modified stimulus-response curve $(\mathrm{p}=0.81)$.

\section{DISCUSSION}

Isometric exercise immediately reduced patellar tendon pain with the effect sustained for at least $45 \mathrm{~min}$. There was a smaller magnitude immediate effect for isotonic exercise that was not sustained. Release of intracortical inhibition was associated with pain reduction and may be implicated as an underlying mechanism for the changes in pain. There was an increase in MVIC postisometric exercise that may be attributed to a decrease in intracortical inhibition. Time course testing of SLDS and the inclusion of a purely baseline measure (no intervention) 1 week prior to the first intervention session demonstrated that changes were due to the intervention, and not TMS or MVIC testing.

The pain reduction observed following isometric exercise may be due to the cortical changes observed and motor neurone pool recruitment, and/or driven by changes at a tissue level. Spinal and supraspinal activation strategies and signals may be different depending on the type of contraction. ${ }^{39}$ The percentage of motor unit activation during an isometric contraction is significantly higher than that during either eccentric or concentric contractions. ${ }^{40}$ These differences may be due to the signal discharged from the spinal cord and/or the central activation of the $\alpha$ (cells bodies located in the ventral horn of the spinal cord that innervate extrafusal muscle fibres) or $\gamma$ (innervate intrafusal muscle fibres found within the muscle spindle) motor neurone pools. ${ }^{41}$ The distinction between isometric contraction and an isotonic contraction is simply whether there is fibre length or whole muscle length change. ${ }^{42}$ During a concentric/eccentric action there must be constant modulation of motor unit activity as less is required in the eccentric phase. There is also greater feedback from muscle spindles during eccentric contractions. ${ }^{43}$ It has been hypothesised that there may be unique activation patterns rather than simply a scaled down version of the activation signal used during the concentric movement related to torque requirements ${ }^{44} 45$ and that there is more cognitive attention required during eccentric actions, especially in planning and movement execution. ${ }^{39}$ However, this is quite complex as 
Figure 3 Individual participant data for single-leg decline squat (SLDS) pre, post and at $45 \mathrm{~min}$ : SLDS pain scores at each interval for individual participants. (A) Isometric intervention and $(B)$ isotonic intervention. Note due to the same pain scores reported for different participants, lines in the isometric intervention graph overlap.
A

Isometric intervention

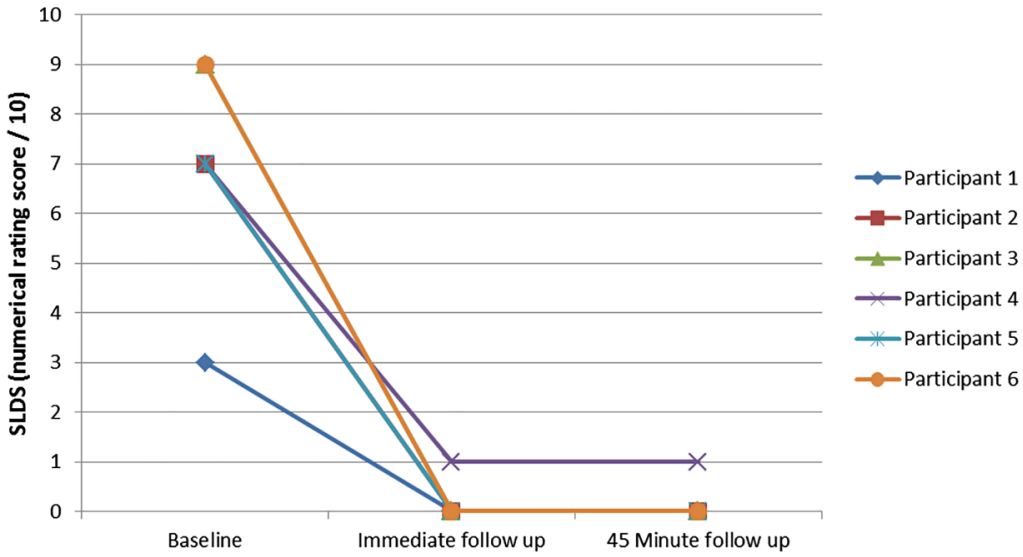

B

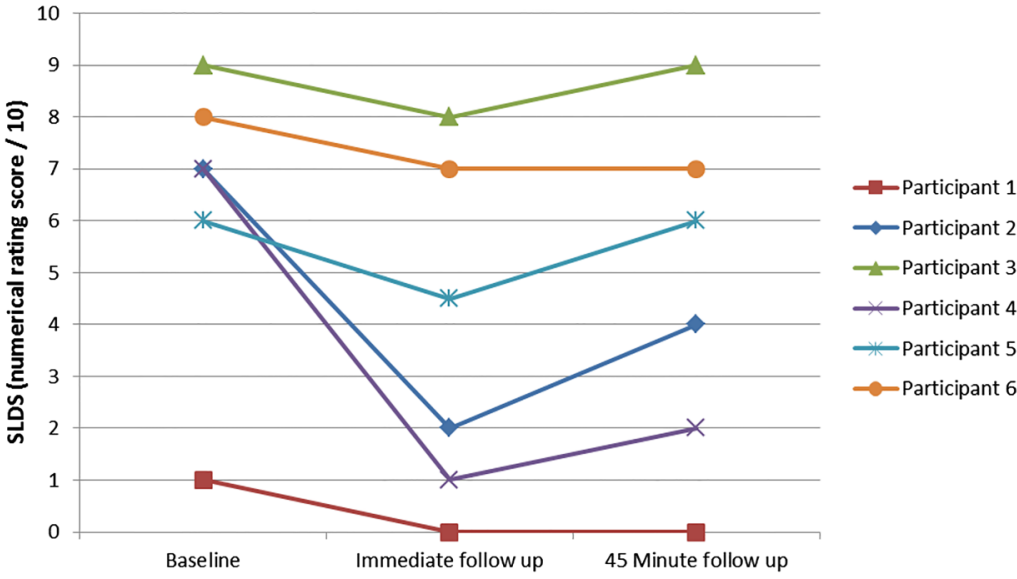

Isotonic intervention

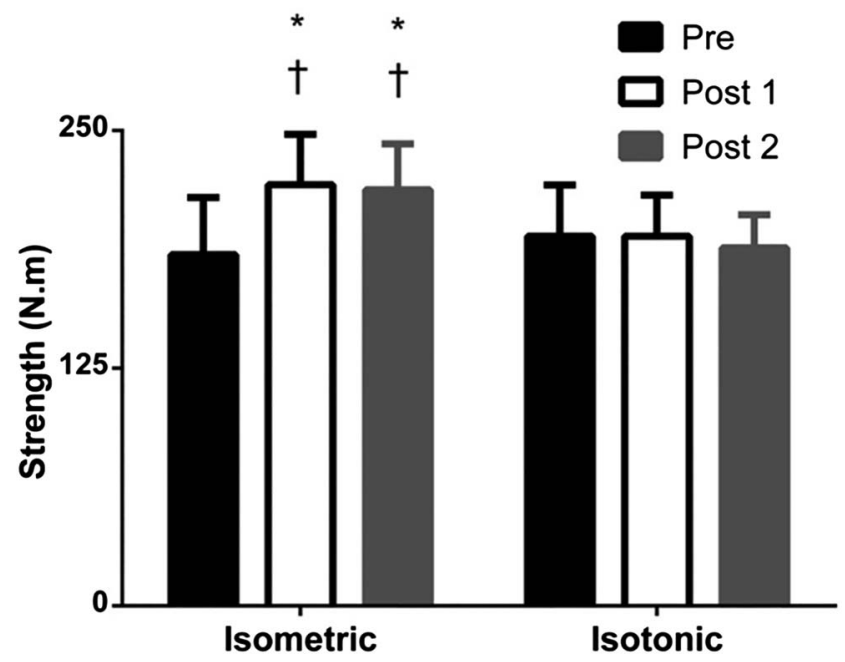

different studies report different size MEPs (representing cortical) and H-reflexes (representing peripheral) depending on contraction type. ${ }^{46}$

Local tissue effects may include changes to cell metabolism, receptor expression and the biochemical environment during isometric exercise, which are then transmitted to the CNS. For example, the balance of ion channel and receptor-operated channel function may change in milliseconds. ${ }^{47}$ This would implicate a different local tissue effect when completing an isometric muscle contraction as compared with an isotonic muscle contraction. A potentially important finding in this unilateral protocol was that there was no effect on the contralateral leg pain in those with bilateral PT $(n=3)$; however, this warrants further investigation with greater numbers. The changes to intracortical inhibition indicate no widespread CNS pain inhibition (a feature following isometric exercise that has been reported in populations without musculoskeletal pain using pressure pain thresholds). The lack of any detectable effect on the contralateral knee pain of those with bilateral pain demonstrates that the analgesia observed on the exercised leg is not simply a consequence of systemic inhibitory control, although it does not rule out this consideration.

Cortical reorganisation reflects changes in the response profile of brain cells. Reorganisation of the motor cortex (M1) can be detected by changes in both the magnitude of the muscle 


\section{$\mathrm{SICl}$}

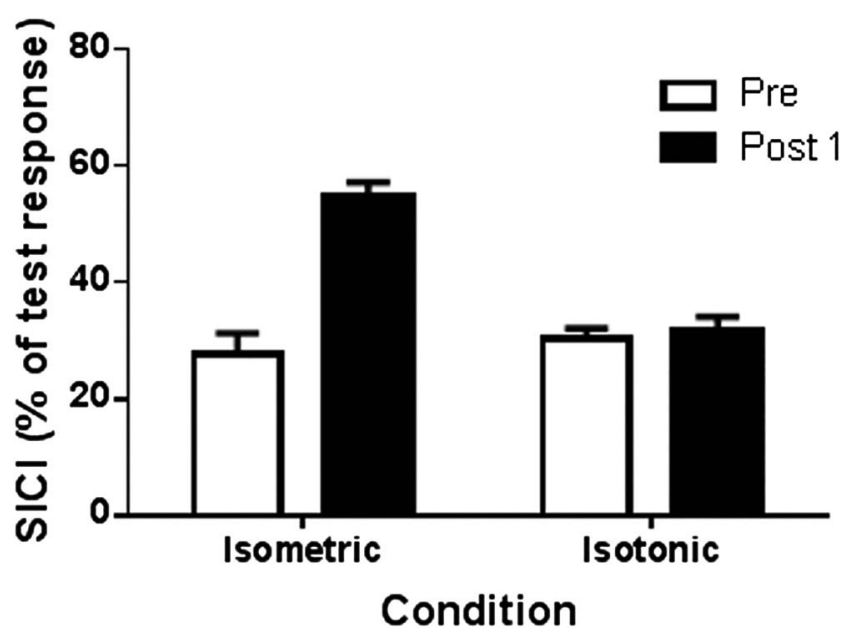

Figure 5 Effect of intervention types on short-interval intracortical inhibition (SICl). There were no differences at baseline in $\mathrm{SICl}$. SICl before and after isometric and isotonic interventions. * Denotes significantly different post isometric from pre isometric $(p<0.05)$; $\dagger$ denotes significant to isotonic group $(p<0.05)$. The lines on the graph represent the range of normal $\mathrm{SICl}$ ration reported for the quadriceps.

response and the cortical topographical motor maps that are evoked by stimulation. Although the changes might occur anywhere along the motor neuroaxis, these are generally attributed to changes in cortical excitability. We did not observe an effect of a single bout of isometric or isotonic contractions on cortical excitability; however, this protocol employed modified stimulusresponse curves. Therefore, it cannot be ruled out that changes were simply not observed using this method. Furthermore, neuroplastic changes to the M1 could require repeated and targeted strategies to address them.

Unlike cortical excitability, SICI appears to be mediated through low-threshold $\mathrm{GABA}_{\mathrm{A}}$ receptor-dependent inhibitory pathways ${ }^{14}$ and was altered with a single bout of isometric exercise. Strength training is known to modulate inhibition in normal controls. ${ }^{48}$ However, few studies have examined intracortical inhibition in other musculoskeletal conditions. In osteoarthritis-related pain, inhibition did not significantly differ from the control group whereas in chronic neuropathic pain, following peripheral nerve lesion, there was a significant reduction in inhibition. ${ }^{49}$ When normal volunteers are subjected to experimental muscle pain SICI levels are increased; ${ }^{28}$ however, the intervention protocol of the current study was non-painful. Despite the limited literature available for comparison, the high levels of inhibition present in people with PT (mean of $27 \%$ whereas data reported for the quadriceps in normal participants ranges between $50 \%$ and $70 \%)^{16}$ appears to be a novel finding. Further, this was altered following a single bout of isometric exercise.

Inhibition affects motor patterning by altering the number of action potentials that reach the corticospinal pathway to activate the motor neurone pool. People with PT appear to be using large amounts of inhibition to moderate their motor output, which may be an aberrant control mechanism compared with normal motor control. This strategy may contribute to the recalcitrance of PT, and may be a new and important consideration in effective rehabilitation. Furthermore, isometric exercise, but not isotonic exercise, modulated inhibition in those with PT, which improves the net corticospinal drive to the motor neurone pool. This change occurred without evidence of a systematic decline in muscle performance (fatigue), as muscle performance (MVIC) was actually improved by the isometric protocol. This difference between the protocols indicates the effect is not simply the result of load or exercise-based analgesia, but is task specific and only observed during isometric contractions. The clinical implications are that isometric exercise using $70 \%$ of MVC may be a useful protocol prior to activity to reduce pain without inducing muscle fatigue. Increased inhibition may be a method of reducing motor recruitment in PT and could be an important finding to address in rehabilitation.

MEPs are reduced during experimental pain conditions. ${ }^{50}$ The testing and intervention protocols here were not painful and this excludes the possibility that the observed effect reflects competitive inhibition or diffuse noxious inhibition.

There are a number of limitations of the study. First, it is acknowledged that the sample size was small. The robustness of the study was improved by the cross-over design and 3-week protocol where sessions were completed on the same day, at the same time of day, with consistent loading prior (and therefore, tendon pain) to the session. However, it is unknown if the results are generalisable given the small numbers. Second, these results are not applicable to people with anterior knee pain and may be specific to PT. The diagnosis of PT is important; it is not expected that positive results would be observed if other presentations of anterior knee pain were included, for example, heavy quadriceps loading may aggravate someone with patellofemoral pain due to the compression in this position. Third, this study only included men, but did include unilateral and bilateral pain presentations. Finally, the inclusion of a non-intervention control group would provide greater quantification of the changes observed.

This study has several clinical implications. Despite the small sample size, participants reported a broad pain severity at baseline reflecting the variability of clinical presentations. Furthermore, there were no non-responders to isometric exercise regardless of pain severity or length of time of symptoms. In contrast, there was variation in the amount of pain reduction following isotonic exercise which was significantly less than from isometric and the effect was not retained at $45 \mathrm{~min}$. Thus, it may be a less attractive pain relief option. The clinical implications are that isometric exercise may be used to reduce pain and motor inhibition in the early stages of rehabilitation and provide an important option for clinicians to offer for painful tendons that are difficult to load. There may be a role for using isometric exercise to reduce motor inhibition and improve responses to strength training as it is currently known that deficits persist despite rehabilitation. ${ }^{51}$ Tendon rehabilitation needs to be progressed beyond isometric exercise to enable a resilient tendon to return to sport. However, this may provide an important option for clinicians to offer in painful tendons that are difficult to load without aggravating symptoms or potentially prestrength training sessions in latter stages.

As a further consideration, the optimum prescription of isometric exercise for pain reduction is not yet known. While this study extensively piloted different protocols prior to data collection, further studies should identify optimal load, length of time under tension, rest and sets. In summary, this is the first study to demonstrate immediate pain reduction using strength training in people with tendinopathy. Future research should continue to investigate exercise for pain relief in other tendons and also provide optimal guidelines around dosage. 


\section{What are the new findings?}

- Heavy isometric exercise immediately reduced patellar tendon pain that was sustained for at least $45 \mathrm{~min}$.

- People with patellar tendinopathy have higher amounts of cortical muscle inhibition for their quadriceps than normal controls.

- Heavy isometric exercise reduced cortical muscle inhibition and may be a factor in the mechanism of pain reduction.

- Isotonic exercise did not result in sustained pain relief or any changes to muscle inhibition.

\section{How might it impact on clinical practice in the near}

future?

- Isometric exercise may be used as analgesia-to reduce pain immediately in patellar tendinopathy.

- Isometric exercise may be useful in-season, preactivity or postactivity, when alternate loading, such as eccentric exercise, has not shown to be beneficial.

- Patellar tendon pain affects muscle inhibition-isometric exercise may be used to reduce pain and change muscle inhibition without a reduction in muscle strength.

Twitter Follow Ebonie Rio at @tendonpain, Jill Cook @ProfjillCook and James Gaida at @tendonresearch

Contributors ER, DK and JC made significant contributions to study design and piloting of the study. CP and JC contributed significantly to protocol design. DK and ER collected the data, and all analysis was completed by ER. All authors made significant contribution to manuscript preparation including editing, key neuroscience concepts and clinical implications according to their specific expertise.

Competing interests $\mathrm{JC}$ was supported by the Australian centre for research into sports injury and its prevention, which is one of the International Research Centres for Prevention of Injury and Protection of Athlete Health supported by the IOC. JC is a NHMRC practitioner fellow (ID 1058493). GLM is supported by an Australian National Health and Medical Research Council Principal Research Fellowship (NHMRC ID 1061279). DK is supported by an Alfred Deakin Post-Doctoral Fellowship. ER is supported by an Australian Postgraduate Award.

Ethics approval Monash University Human Ethics.

Provenance and peer review Not commissioned; externally peer reviewed.

Data sharing statement Silent periods have not been included due to the word limit; this may be accessed by contacting the corresponding author.

\section{REFERENCES}

1 Frohm A, Saartok T, Halvorsen K, et al. Eccentric treatment for patellar tendinopathy: a prospective randomised short-term pilot study of two rehabilitation protocols. Br J Sports Med 2007:41:e7.

2 Maffulli N, Walley G, Sayana MK, et al. Eccentric calf muscle training in athletic patients with Achilles tendinopathy. Disabil Rehabil 2008; 30:1677-84

3 Visnes $\mathrm{H}$, Bahr R. The evolution of eccentric training as treatment for patellar tendinopathy (jumper's knee): a critical review of exercise programmes. $\mathrm{Br}$ J Sports Med 2007;41:217-23.

4 Woodley BL, Newsham-West RJ, Baxter GD. Chronic tendinopathy: effectiveness of eccentric exercise. Br J Sports Med 2007;41:188-98; discussion 199.

5 Alfredson $\mathrm{H}$, Pietila $\mathrm{T}$, Jonsson $\mathrm{P}$, et al. Heavy-load eccentric calf muscle training for the treatment of chronic Achilles tendinosis. Am J Sports Med 1998;26:360-6.

6 Cook JL, Purdam CR. The challenge of managing tendinopathy in competing athletes. Br J Sports Med 2014;48:506-9.

7 Visnes $\mathrm{H}$, Hoksrud A, Cook J, et al. No effect of eccentric training on jumper's knee in volleyball players during the competitive season: a randomized clinical trial. Clin J Sport Med 2005;15:227-34.
8 Fredberg U, Bolvig L, Andersen NT. Prophylactic training in asymptomatic soccer players with ultrasonographic abnormalities in Achilles and patellar tendons: the Danish Super League Study. Am J Sports Med 2008;36:451-60.

9 Jonsson $\mathrm{P}$, Alfredson $\mathrm{H}$. Superior results with eccentric compared to concentric quadriceps training in patients with jumper's knee: a prospective randomised study. Br J Sports Med 2005:39:847-50.

10 Kongsgaard $M$, Kovanen V, Aagaard $P$, et al. Corticosteroid injections, eccentric decline squat training and heavy slow resistance training in patellar tendinopathy. Scand J Med Sci Sports 2009;19:790-802.

11 Kosek E, Ekholm J. Modulation of pressure pain thresholds during and following isometric contraction. Pain 1995:61:481-6.

12 Koltyn KF, Umeda M. Contralateral attenuation of pain after short-duration submaximal isometric exercise. J Pain 2007:8:887-92.

13 Tsao H, Danneels LA, Hodges PW. ISSLS prize winner: smudging the motor brain in young adults with recurrent low back pain. Spine 2011;36:1721-7.

14 Ziemann U. Pharmacology of TMS. Supp/ Clin Neurophysio/ 2003;56:226-31.

15 Kobayashi M, Hamada T, Kogo M, et al. Developmental profile of GABAA-mediated synaptic transmission in pyramidal cells of the somatosensory cortex. Eur I Neurosci 2008:28:849-61.

16 Goodwill AM, Pearce AJ, Kidgell DJ. Corticomotor plasticity following unilateral strength training. Muscle Nerve 2012;46:384-93.

17 Pitman BM, Semmler JG. Reduced short-interval intracortical inhibition after eccentric muscle damage in human elbow flexor muscles. J App/ Physiol 2012:113:929-36.

18 Dean BJ, Gwilym SE, Carr AJ. Why does my shoulder hurt? A review of the neuroanatomical and biochemical basis of shoulder pain. Br J Sports Med 2013;47:1095-104

19 Schulz KF, Grimes DA. Allocation concealment in randomised trials: defending against deciphering. Lancet 2002;359:614-18.

20 Visentini PJ, Khan KM, Cook JL, et al. The VISA score: an index of severity of symptoms in patients with jumper's knee (patellar tendinosis). Victorian Institute of Sport Tendon Study Group. J Sci Med Sport 1998;1:22-8.

21 Purdam C, Cook J, Hopper D, et al. Discriminative ability of functional loading tests for adolescent jumper's knee. Phys Ther Sport 2003;4:3-9.

22 Wilson SA, Thickbroom GW, Mastaglia FL. Topography of excitatory and inhibitory muscle responses evoked by transcranial magnetic stimulation in the human moto cortex. Neurosci Lett 1993:154:52-6.

23 Pearce AJ, Clark RA, Kidgell DJ. A comparison of two methods in acquiring stimulus-response curves with transcranial magnetic stimulation. Brain Stimul 2013:6:306-9

24 Carroll TJ, Riek S, Carson RG. Reliability of the input-output properties of the cortico-spinal pathway obtained from transcranial magnetic and electrical stimulation. J Neurosci Methods 2001;112:193-202.

25 Reis J, Swayne OB, Vandermeeren Y, et al. Contribution of transcranial magnetic stimulation to the understanding of cortical mechanisms involved in motor control. J Physiol 2008:586:325-51.

26 Garry MI, Thomson RH. The effect of test TMS intensity on short-interval intracortical inhibition in different excitability states. Exp Brain Res 2009:193:267-74.

27 Ilic TV, Meintzschel F, Cleff U, et al. Short-interval paired-pulse inhibition and facilitation of human motor cortex: the dimension of stimulus intensity. J Physiol 2002;545(Pt 1):153-67.

28 Chipchase L, Schabrun S, Cohen L, et al. A checklist for assessing the methodological quality of studies using transcranial magnetic stimulation to study the motor system: an international consensus study. Clin Neurophysiol 2012;123:1698-704.

29 Reider B, Marshall JL, Koslin B, et al. The anterior aspect of the knee joint. J Bone Joint Surg Am 1981;63:351-6.

30 Carroll TJ, Riek S, Carson RG. The sites of neural adaptation induced by resistance training in humans. J Physiol 2002;544(Pt 2):641-52.

31 Ahtiainen JP, Pakarinen A, Alen $\mathrm{M}$, et al. Muscle hypertrophy, hormonal adaptations and strength development during strength training in strength-trained and untrained men. Eur J Appl Physiol 2003:89:555-63.

32 Hendy AM, Spittle M, Kidgell DJ. Cross education and immobilisation: mechanisms and implications for injury rehabilitation. J Sci Med Sport 2012;15:94-101.

33 Kidgell DJ, Pearce AJ. Corticospinal properties following short-term strength training of an intrinsic hand muscle. Hum Mov Sci 2010;29:631-41.

34 Kidgell DJ, Stokes MA, Pearce AJ. Strength training of one limb increases corticomotor excitability projecting to the contralateral homologous limb. Motor Control 2011:15:247-66.

35 Latella C, Kidgell DJ, Pearce AJ. Reduction in corticospinal inhibition in the trained and untrained limb following unilateral leg strength training. Eur J Appl Physiol 2012:112:3097-107.

36 Weier AT, Pearce AJ, Kidgell DJ. Strength training reduces intracortical inhibition. Acta Physiol (Oxf) 2012;206:109-19.

37 Selvanayagam VS, Riek S, Carroll TJ. A systematic method to quantify the presence of cross-talk in stimulus-evoked EMG responses: implications for TMS studies. J App/ Physiol 2012;112:259-65 


\section{Original article}

38 Lentz $\mathrm{M}$, Nielsen JF. Post-exercise facilitation and depression of $\mathrm{M}$ wave and motor evoked potentials in healthy subjects. Clin Neurophysiol 2002;113:1092-8.

39 Duchateau J, Enoka RM. Neural control of shortening and lengthening contractions: influence of task constraints. J Physiol 2008;586(Pt 24):5853-64.

40 Babault N, Pousson M, Ballay Y, et al. Activation of human quadriceps femoris during isometric, concentric, and eccentric contractions. J App/ Physiol 2001;91:2628-34.

41 Tax AA, Denier van der Gon JJ, Gielen CC, et al. Differences in central control of m. biceps brachii in movement tasks and force tasks. Exp Brain Res 1990;79:138-42.

42 Ishikawa M, Komi PV, Grey MJ, et al. Muscle-tendon interaction and elastic energy usage in human walking. J Appl Physiol 2005;99:603-8.

43 Hulliger $M$, Nordh E, Vallbo AB. Discharge in muscle spindle afferents related to direction of slow precision movements in man. J Physiol 1985;362:437-53.

44 Enoka RM. Eccentric contractions require unique activation strategies by the nervous system. J Appl Physiol 1996;81:2339-46.

45 Nardone A, Schieppati M. Shift of activity from slow to fast muscle during voluntary lengthening contractions of the triceps surae muscles in humans. J Physiol 1988;395:363-81.
46 Abbruzzese $G$, Morena $M$, Spadavecchia $L$, et al. Response of arm flexor muscles to magnetic and electrical brain stimulation during shortening and lengthening tasks in man. J Physiol 1994;481(Pt 2):499-507.

47 Hudspith M, Siddall P, Munglani R. Physiology of pain. In: Hemmings H, Hopkins P, eds. Foundations of anesthesia. 2nd edn. Mosby Elsevier, 2006:267-85.

48 Aagaard P, Simonsen EB, Andersen JL, et al. Neural inhibition during maximal eccentric and concentric quadriceps contraction: effects of resistance training. J App/ Physiol (1985) 2000;89:2249-57.

49 Schwenkreis $\mathrm{P}$, Scherens $\mathrm{A}$, Ronnau AK, et al. Cortical disinhibition occurs in chronic neuropathic, but not in chronic nociceptive pain. BMC Neurosci 2010;11:73.

50 Le Pera D, Graven-Nielsen T, Valeriani M, et al. Inhibition of motor system excitability at cortical and spinal level by tonic muscle pain. Clin Neurophysiol 2001;112:1633-41.

51 Paavola M, Kannus P, Paakkala T, et al. Long-term prognosis of patients with Achilles tendinopathy. An observational 8-year follow-up study. Am I Sports Med 2000;28:634-42. 